\title{
FUSÃO INTERSOMÁTICA LOMBAR TRANSFORAMINAL: EXPERIÊNCIA DE UMA INSTITUIÇÃO
}

\author{
TRANSFORAMINAL LUMBAR INTERBODY FUSION: A SINGLE-CENTER EXPERIENCE \\ FUSIÓN INTERSOMÁTICA LUMBAR TRANSFORAMINAL: \\ LA EXPERIENCIA DE UNA INSTITUCIÓN
}

Vinicius de Meldau Benites ${ }^{1}$, Eduardo Augusto lunes ${ }^{1}$, Alexandre José dos Reis Elias ${ }^{1}$, Rodrigo Miziara Yunes ${ }^{1}$,

Franz Joojl Onishi', Sérgio Cavalheiro ${ }^{2}$

RESUMO

Objetivo: Relatar a experiência inicial de um serviço de referência em cirurgia da coluna em São Paulo, Brasil com a técnica de fusão intersomática lombar transforaminal (TLIF) nas suas mais variadas indicações. Métodos: Avaliamos retrospectivamente os dados gravados em prontuário de 25 pacientes que foram submetidos à cirurgia com a técnica de TLIF no ano de 2011. Um paciente foi excluído porque não consideramos que a TLIF foi a principal técnica empregada. As indicações incluíram nove casos de hérnia de disco lombar, sete espondilolisteses, quatro cirurgias de revisão, sendo duas por pseudoartrose e duas por lombalgias e, finalmente, quatro pacientes com estenose espinhal lombar. Resultados: Todos os pacientes referiram melhora da dor e/ou claudicação neurológica em comparação com o estado pré-operatório. Apenas cinco pacientes continuaram usando alguma medicação analgésica. Cinco pacientes apresentaram alguma complicação, mas somente duas delas estão relacionadas diretamente com o procedimento. Conclusões: Trata-se de uma técnica segura, possível de ser realizada em todos os níveis da coluna lombar e aplicável a grande parte das doenças que acometem essa região da coluna.

Descritores: Fusão vertebral; Espondilolistese; Dor lombar; Estenose espinal; Região lombossacral.

\begin{abstract}
Objective: Describe the early results and experience from a reference center in spine surgery in São Paulo, Brazil with transforaminal lumbar interbody fusion (TLIF) technique in its various indications. Methods: We retrospectively reviewed the medical records of 25 patients who underwent surgery with TLIF technique in 2011. One patient was excluded because we considered that TLIF was not the most important technique used. The indications were 9 lumbar disc herniations, 7 spondylolistheses, 4 revision surgeries of which 2 were for pseudoarthroses and 2 for low back pain, and finally, 4 lumbar spinal stenoses. Results: All the patients reported low back pain and/or neurologic claudication improvement when comparing to preoperative status. Only five patients continued using analgesics. Five patients presented some complication, but only two of them were related to the procedure. Conclusions: TLIF is a safe technique which can be performed at any lumbar level of the lumbar spine and is applicable to the majority of diseases that affect this region.
\end{abstract}

Keywords: Spinal fusion; Spondylolisthesis; Low back pain; Spinal stenosis; Lumbosacral region.

RESUMEN

Objetivo: Relatar la experiencia de un servicio de referencia en cirugía de la columna en São Paulo, Brasil con la técnica de fusión intersomática lumbar transforaminal (TLIF) en sus más variables indicaciones. Métodos: Valoramos retrospectivamente el historial médico de 25 pacientes que fueron sometidos a la cirugía con la técnica de TLIF en el año de 2011. Un paciente fue excluido porque no consideramos que la TLIF fue la principal técnica empleada. Las indicaciones incluyeron nueve casos de hernia de disco lumbar, siete espondilolistesis, cuatro cirugías de revisión siendo dos por pseudoartrosis y dos por lumbalgias y, finalmente, cuatro pacientes con estenosis espinal. Resultados: Todos los pacientes presentaron mejora del dolor y/o claudicación neurogénicas en comparación con el status preoperatorio. Solamente cinco pacientes continuaron usando alguna medicación analgésica. Cinco pacientes presentaran alguna complicación, pero sólo dos de ellas están relacionadas directamente al procedimiento. Conclusiones: Es una técnica segura, posible de ser realizada en todos los niveles de la columna lumbar y es aplicable a la mayoría de las enfermedades que afectan a esta región de la columna.

Descriptores: Fusión vertebral; Espondilolistesis; Dolor de la región lumbar; Estenosis espinal; Región lumbosacra.

\section{INTRODUÇÃO}

Desde que foi inicialmente publicada por Harms and Jeszenszky em 1998 a técnica cirúrgica do TLIF e a série de 191 casos operados $^{1}$, inúmeros outros trabalhos abordando suas indicações, complicações, séries de casos e comparações com outras técnicas cirúrgicas surgiram. As vantagens em relação a outras técnicas cirúrgicas que incluem a fusão intersomática lombar posterior (PLIF) e a anterior (ALIF) e a vasta gama de indicações, fizeram o TLIF ganhar popularidade entre os cirurgiões de coluna.

As principais vantagens em relação ao ALIF incluem a fusão intersomática sem a necessidade do acesso ventral, seja ele transperitoneal ou retroperitoneal, o que em outras palavras significa menor agressão cirúrgica ao paciente, além de não haver o risco de lesão do plexo simpático que pode acarretar ejaculação retrógrada nos homens ${ }^{2,3}$.

\footnotetext{
1. Neurocirurgião, Médico Assistente do Grupo de Coluna da Disciplina de Neurocirurgia da Universidade Federal de São Paulo - Escola Paulista de Medicina , São Paulo, SP, Brasil.
} 2. Neurocirurgião, ProfessorTitular de Neurocirurgia da Universidade Federal de São Paulo - Escola Paulista de Medicina, São Paulo, SP, Brasil. 
Em relação ao PLIF as vantagens relacionam-se a descompressão das estruturas neurais através da ampliação do forame intervertebral lombar, além da menor perda sanguínea principalmente quando essas técnicas são comparadas em cirurgias de múltiplos níveis. A mobilização das estruturas neurais durante a realização do PLIF pode resultar em aracnoidite, radiculopatia e maior propensão à fístula liquórica, quando comparado com o TLIF. Por fim, a técnica de TLIF pode ser utilizada em todos os níveis lombares, já que não retrai a dura-máter e consequentemente o cone medular nos níveis superiores, ao contrário do que ocorre no PLIF que pode ser indicado apenas de $\mathrm{L} 3$ a S13-5.

Baseado nas vantagens que tal técnica oferece tanto ao cirurgião, quanto ao paciente, passamos a adotá-la em nosso serviço para a maioria dos casos em que antes era utilizada apenas a artrodese póstero-lateral e mostramos a nossa série de casos no último ano.

\section{MÉTODOS}

No período de janeiro de 2011 a janeiro de 2012 foram operados 25 pacientes, sendo 13 homens e 12 mulheres, utilizando-se a técnica de TLIF, porém um paciente do sexo masculino foi excluído por se tratar de instrumentação tóraco-sacral de T10-S1 e apenas TLIF em um nível. As indicações incluíram espondilolistese degenerativa e lítica pseudoartrose pós artrodese posterolateral, estenose espinhal lombar, hérnia de disco lombar e lombalgia refratária ao tratamento clínico. Os autores realizavam artrodese posterolateral ou PLIF rotineiramente, até serem introduzidos ao procedimento de TLIF.

Dados referentes à idade, sexo, diagnóstico, tempo de cirurgia, tempo de internação, perda sanguínea, evolução pós-operatória e complicações foram coletados para cada procedimento. Em vista do curto período de seguimento pós-cirúrgico, alguns dados são apenas descritivos com relação a nossa experiência e comparativos com a literatura.

O procedimento de fusão intersomática lombar transforaminal é realizado com o paciente em decúbito ventral sobre coxins de forma a haver mínima flexão de quadris e joelhos. A exposição posterior da coluna é realizada de modo padrão, com atenção para a preservação dos ligamentos supra e interespinhosos. Os locais para a colocação dos parafusos pediculares é preparado e os mesmos são introduzidos. O uso de intensificador de imagem é importante para assegurarmos o bom posicionamento do material de instrumentação.

A pars interarticularis de cada nível selecionado é removida e a hemifacetectomia das facetas superior e inferior é realizada. 0 lado escolhido é o esquerdo nos casos em que não há sintomas radiculares ou déficits neurológicos, caso contrário, optamos por expor o forâmen correspondente ao lado sintomático. Nos casos de espondilolistese, em geral fazemos a artrectomia bilateral para facilitar a correção da deformidade. A raiz nervosa do nível é visualizada na região ínfero-medial do pedículo da vértebra superior e a raiz do nível abaixo é protegida superior e medialmente ao pedículo da vértebra inferior. Após a exposição do disco intervertebral, é feito uma abertura lateral no ânulo com o bisturi, seguido da retirada do conteúdo discal com o uso de curetas e pinças para nucleotomia. Por fim realizamos a decorticação do platô vertebral superior e inferior.

A técnica de distração do espaço intervertebral é realizada com a colocação de haste longitudinal contralateral ao procedimento seguida de distração, e por vezes utilizamos distratores nos parafusos pediculares ipsilateralmente ao procedimento. Preenchemos os dois terços anteriores com enxerto ósseo autólogo e em seguida posicionamos uma gaiola intersomática tipo banana preenchida por osso. A distração é solta e as hastes moldadas são fixadas aos parafusos sob moderada compressão para favorecer a lordose.

Finalmente fazemos a decorticação das estruturas ósseas posterolaterais assim como das lâminas e colocamos enxerto ósseo autólogo misturado a enxerto ósseo sintético. É instalado sistema de drenagem por contra-abertura na pele e realizado o fechamento por planos e sutura intradérmica na pele.

\section{RESULTADOS}

Os 24 pacientes considerados para este estudo possuíam entre 25 e 70 anos com a média de 50,2 anos. O tempo de internação variou de 3 a 15 dias, com média de 5,1 dias. A perda sanguínea variou de 200 a $1600 \mathrm{ml}$, com média de 520ml, tendo sido necessário hemotransfusão em 25\% dos pacientes no intraoperatório. Em 19 pacientes foi realizado TLIF em apenas um nível com duração da cirurgia tendo variado de 2,5 a 4 horas, quatro pacientes foram submetidos a TLIF em dois níveis com o tempo cirúrgico variando de 3 a 4,5 horas e em apenas um paciente realizamos TLIF em três níveis, tendo a cirurgia 4,5 horas de duração. No total foram realizados 30 TLIF's.

Não houve complicações cirúrgicas intraoperatórias. Tivemos cinco pacientes que apresentaram alguma complicação perioperatória, porém consideramos que apenas duas delas foram diretamente relacionadas ao ato cirúrgico. Um paciente (paciente 3) sofreu broncoespasmo durante a intubação orotraqueal, que foi prontamente revertida. Um paciente evoluiu com pneumonia hospitalar (paciente 1), necessitando o uso de antibiótico intravenoso o que prolongou sua permanência hospitalar. Houve um caso de infecção de ferida operatória (paciente 6) que surgiu após a alta hospitalar e foi tratado com antibiótico via oral com bom resultado. Um paciente (paciente 15) apresentou radiculopatia que não era exacerbada pela manobra de Lasègue, e foi tratado com pré-gabalina com remissão do quadro álgico.Por fim tivemos uma complicação de maior gravidade (paciente 18), uma paciente que sofreu hemotórax durante a punção de artéria subclávia esquerda para acesso venoso central e necessitou de drenagem pleural e hemotransfusão na Unidade de Terapia Intensiva, mas teve alta hospitalar em bom estado geral, apesar do maior tempo de internação.

Os diagnósticos pré-operatórios, assim como outros dados relacionados aos pacientes estão relacionados na Tabela 1. Com relação ao seguimento pós-operatório não observamos piora do quadro de lombalgia em comparação ao pré-operatório em nenhum paciente. Apenas cinco pacientes ainda referem dor lombar

Tabela 1. Resumo dos dados dos pacientes.

\begin{tabular}{c|c|c|c|c}
\hline $\mathbf{N}^{0}$ & Idade/Sexo & Diagnóstico & Tipo de cirurgia & $\begin{array}{c}\text { Tempo de } \\
\text { internação }\end{array}$ \\
\hline 1 & $63 / \mathrm{M}$ & Estenose espinhal lombar & TLIF L3-L4, L4-L5, L5-S1 & 10 \\
\hline 2 & $64 / F$ & HNP L4-L5, L5-S1 & $\begin{array}{c}\text { TLIF L4-L5, L5-S1. Instrumentação } \\
\text { L3-S1 }\end{array}$ & 4 \\
\hline 3 & $60 / F$ & $\begin{array}{c}\text { Estenose espinhal lombar + } \\
\text { listese Gl }\end{array}$ & TLIF L4-L5. Instrumentação L4-S1 & 6 \\
\hline 4 & $57 / M$ & Listese L5-S1 GII & TLIF L5-S1. Instrumentação L4-S1 & 4 \\
\hline 5 & $56 / M$ & Listese L4-L5 pós discite & TLIF L4-L5. Instrumentação L3-S1 & 15 \\
\hline 6 & $63 / F$ & Listese L3-L4 & TLIF L3-L4 & 4 \\
\hline 7 & $37 / F$ & Pseudoartrose & TLIF L5-S1 & 3 \\
\hline 8 & $41 / F$ & HNP L4-L5 & TLIF L4-L5 & 3 \\
\hline 9 & $70 / M$ & HNP L5-S1 & TLIF L5-S1 & 3 \\
\hline 10 & $39 / M$ & Pseudoartrose & TLIF L5-S1 & 3 \\
\hline 11 & $62 / M$ & Estenose espinhal lombar & TLIF L4-L5 & 3 \\
\hline 12 & $66 / M$ & Listese G1 & TLIF L4-L5 & 3 \\
\hline 13 & $50 / M$ & HNP L4-L5 & TLIF L4-L5 & 4 \\
\hline 14 & $40 / F$ & DDD L3-L4, HNP L4-L5 & TLIF L4-L5.Instrumentação L3-L5 & 7 \\
\hline 15 & $44 / F$ & DDD L4-L5, HNP L5-S1 & TLIF L5-S1.Instrumentação L4-S1 & 5 \\
\hline 16 & $25 / F$ & HNP L5-S1 & TLIF L5-S1 & 4 \\
\hline 17 & $41 / M$ & Listese L4-L5 & TLIF L4-L5 & 6 \\
\hline 18 & $47 / F$ & Lombalgia (revisão) & TLIF L4-S1 & 13 \\
\hline 19 & $57 / M$ & Lombalgia (revisão) & TLIF L4-S1 & 5 \\
\hline 20 & $52 / M$ & HNP L5-S1 & TLIF L5-S1 & 4 \\
\hline 21 & $44 / F$ & Listese L4-L5 & TLIF L4-L5 & 4 \\
\hline 22 & $31 / F$ & HNP L4-L5, L5-S1 & TLIF L4-S1 & 3 \\
\hline 23 & $35 / F$ & Listese L5-S1 & TLIF L5-S1 & 4 \\
\hline 24 & $61 / M$ & Estenose espinhal lombar & TLIF L3-L4 & 4 \\
\hline HNP- hérnia de núcleo pulposo; G - grau; DDD - doença degenerativa discal. & \\
\hline
\end{tabular}


e necessitam do uso de alguma medicação para analgesia. Os quatro pacientes que tinham como queixa principal a claudicação neurológica intermitente evoluíram com resolução dos sintomas. É necessário maior tempo de seguimento para um detalhamento maior dessas informações.

\section{DISCUSSÃO}

O grupo de problemas da região lombo-sacra que podem ser tratados com a técnica de TLIF incluem a instabilidade segmentar com lombalgia refratária ao tratamento clínico, hérnia de disco com instabilidade e lombociatalgia, espondilolistese degenerativa com queixas álgicas crônicas ou associada à claudicação neurológica intermitente, espondilolistese displásica ou lítica associado à lombalgia ou lombociatalgia ${ }^{3,6,7}$, escoliose degenerativa ${ }^{8}$ e estenose de canal espinhal associada a queixas neurológicas incluindo claudicação neurológica intermitente e nos casos de reoperação de disco intervertebral consequente a lombalgia ou lombociatalgia intratável clinicamente ${ }^{1}$. Tais indicações foram respeitadas em nosso grupo.

As contra-indicações incluem os casos de cifose lombar fixa, anomalias do forame intervertebral e casos relacionados a problemas psicossociais ou de ganho secundário ${ }^{1}$.

Os dados biomecânicos relatados na literatura conferem maior estabilidade aos sistemas que associam a artrodese póstero-lateral à fusão intersomática por qualquer técnica. A comparação efetiva das duas técnicas mais difundidas, PLIF e TLIF, demonstraram que elas são equivalentes no que se refere à rigidez da construção do sistema e ao range of motion ${ }^{4}$.

Devido à grande estabilidade que o sistema de fusão $360^{\circ}$ (posterior e intersomática) proporciona imediatamente após o aperto da instrumentação, não há a necessidade do uso de órteses. Sendo assim, o paciente é estimulado à mobilização ativa precoce, como caminhar e sentar-se, o que resulta em menor tempo de internação hospitalar. O retorno ao trabalho deve ser o mais rápido quanto possível.

A técnica cirúrgica que nós utilizamos é a preconizada por Harms, porém na maioria das vezes não contamos com o Harms cage e utilizamos gaiolas tipo banana de material biocompatível e inabsorvível (polieteretercetona - PEEK). Smith et al. ${ }^{9}$ descrevem um índice de até 18,2\% de não união intersomática com o uso de gaiolas bioabsorvíveis, além de estarem sujeitos a grande risco de migração. Por outro lado, Coe atesta a eficácia de gaiolas bioabsorvíveis preenchidas com enxerto autólogo de crista ilíaca ${ }^{10}$ A colocação de uma única gaiola não confere maior mobilidade à construção ${ }^{11}$.

A distração mecânica que é realizada na técnica de TLIF favorece a reposição das estruturas da coluna vertebral nos casos de deformidades segmentares e auxilia o restabelecimento do balanço sagital nas demais patologias. Favorece a preservação de estruturas importantes do ponto de vista biomecânico como o ligamento amarelo e o interespinhoso, assim como da raiz nervosa e dura-máter adjacente ${ }^{1}$.

A técnica minimamente invasiva para a realização de TLIF está em franco desenvolvimento e os resultados apresentados apontam para taxa de fusão equivalente à técnica aberta, porém sem clara diminuição no índice de complicações ${ }^{8,12-15}$.

As complicações cirúrgicas que ocorreram em nosso grupo de pacientes, no que se refere inclusive à incidência, estão descritas na literatura. Tormenti et al. ${ }^{16}$ ressaltam que maior atenção deve ser tomada nas cirurgias de revisão, pois a taxa de complicações é significativamente maior do que nas cirurgias primárias.

O nosso seguimento pós-operatório variou de 3 a 12 meses, e nesse curto período, não observamos piora da dor em nenhum paciente, sendo que todos se mostraram satisfeitos com o resultado cirúrgico. Tal análise está de acordo com as recentes publicações dos mais diversos centros de coluna ${ }^{17-20}$.

\section{CONCLUSÃO}

A fusão intersomática lombar transforaminal é uma técnica segura, efetiva e pode ser utilizada em grande parte das doenças que acometem a coluna lombar, independente da idade. É superior a outras técnicas de fusão interssomática sob os mais variados aspectos, porém ressaltamos o baixo risco de lesão neurológica quando comparado a fusão intersomática lombar posterolateral e de não haver necessidade de acesso anterior seguido de acesso posterior como na fusão intersomática lombar anterior.

\section{REFERÊNCIAS}

1. Harms J, Jeszenszky D. Die posteriore, lumbale, interkorporelle Fusion in unilateraler transforaminaler Tech Oper Orthop Traumatol. 1998;10:90-102.

2. Hee HT, Castro FP Jr, Majd ME, Holt RT, Myers L. Anterior/posterior lumbar fusion versus transforaminal lumbar interbody fusion: analysis of complications and predictive factors. J Spinal Disord. 2001;14(6):533-40.

3. Kim JS, Lee KY, Lee SH, Lee HY. Which lumbar interbody fusion technique is better in terms of level for the treatment of unstable isthmic spondylolisthesis? J Neurosurg Spine. 2010;12(2):171-7.

4. Ames PC, Acosta FL, Chi J, lyengar J, Muiru W, Acaroglu E, et al. Biomechanical comparison of posterior lumbar interbody fusion and transforaminal lumbar interbody fusion performed at 1 and 2 levels. Spine. 2005;30(19):E562-566

5. Humphreys SC, Hodges SD, Patwardhan AG, Eck JC, Murphy RB, Covington LA. Comparison of posterior and transforaminal approaches to lumbar interbody fusion. Spine (Phila Pa 1976). 2001:26(5):567-71.

6. Lauber S, SchulteTL, Liljenqvist U, Halm H, Hackenberg L. Clinical and radiologic 2-4-year results of transforaminal lumbar interbody fusion in degenerative and isthmic spondylolisthesis grades 1 and 2. Spine (Phila Pa 1976).2006:31(15):1693-8.

7. Goyal N, Wimberley DW, Hyatt A, Zeiller S, Vaccaro AR, Hilibrand AS, et al. Radiographic and clinical outcomes after instrumented reduction and transforaminal lumbar interbody fusion of mid and high-grade isthmic spondylolisthesis. J Spinal Disord Tech. 2009:22(5):321-7.

8. Karikari IO, Grossi PM, Nimjee SM, Hardin C, Hodges TR, Hughes BD, et al. Minimally invasive lumbar interbody fusion in patients older than 70 years of age: analysis of periand postoperative complications. Neurosurgery. 2011;68(4):897-902.

9. Smith AJ, Arginteanu M, Moore F, Steinberger A, Camins M. Increased incidence of cage migration and nonunion in instrumented transforaminal lumbar interbody fusion with bioabsorbable cages. J Neurosurg Spine. 2010;13(3):388-93.

10. Coe JD. Instrumented transforaminal lumbar interbody fusion with bioabsorbable polymer implants and iliac crest autograft. Neurosurg Focus. 2004;16(3):E11.

11. Harris BM, Hilibrand AS, Savas PE, Pellegrino A, Vaccaro AR, Siegler S, et al. Transfo-

raminal lumbar interbody fusion: the effect of various instrumentation techniques on the flexibility of the lumbar spine. Spine (Phila Pa 1976). 2004;29(4):E65-70

12. Wu RH, Fraser JF, Härtl R. Minimal access versus open transforaminal lumbar interbody fusion: meta-analysis of fusion rates. Spine (Phila Pa 1976). 2010;35(26):2273-81.

13. Holly LT, Schwender JD, Rouben DP, Foley KT. Minimally invasive transforaminallumbar interbody fusion: indications, technique, and complications. Neurosurg Focus. 2006:20(3):E6

14. Shunwu F, Xing Z, Fengdong Z, Xiangqian F. Minimally invasive transforaminal lumbar interbody fusion for the treatment of degenerative lumbar diseases. Spine (Phila Pa 1976). 2010;35(17):1615-20

15. Adogwa O, Parker SL, Bydon A, Cheng J, McGirt MJ. Comparative effectiveness of minimally invasive versus open transforaminal lumbar interbody fusion: 2-year assessment of narcotic use, return to work, disability, and quality of life. J Spinal Disord Tech. 2011:24(8):479-84.

16. Tormenti MJ, Maserati MB, Bonfield CM, Gerszten PC, Moossy JJ, Kanter AS, et al. Perioperative surgical complications of transforaminal lumbar interbody fusion: a single-center experience. J Neurosurg Spine. 2012;16(1):44-50.

17. Parker SL, Adogwa O, Paul AR, Anderson WN, Aaronson O, Cheng JS, et al. Utility of minimum clinically important difference in assessing pain, disability, and health state after transforaminal lumbar interbody fusion for degenerative lumbar spondylolisthesis. J Neurosurg Spine. 2011;14(5):598-604

18. Jagannathan J, Sansur CA, Oskouian RJ Jr, Fu KM, Shaffrey Cl. Radiographic restoration of lumbar alignment after transforaminal lumbar interbody fusion. Neurosurgery. 2009:64(5):955-63.

19. Rosenberg WS, Mummaneni PV. Transforaminal lumbar interbody fusion: technique, complications, and early results. Neurosurgery. 2001;48(3):569-74;

20. Hackenberg L, Halm H, Bullmann V, Vieth V, Schneider M, Liljenqvist U. Transforaminal lumbar interbody fusion: a safe technique with satisfactory three to five year results. Eur Spine J. 2005;14(6):551-8 